\title{
Mechanisms underlying the perifocal neuroprotective effect of the Nrf2-ARE signaling pathway after intracranial hemorrhage
}

This article was published in the following Dove Press journal:

Drug Design, Development and Therapy

17 November 2015

Number of times this article has been viewed

\author{
Xiao-ping Yin ${ }^{1,2}$ \\ Zhi-ying Chen ${ }^{2}$ \\ Jun Zhou' \\ Dan Wu ${ }^{1,3}$ \\ Bing $\mathrm{Bao}^{2}$ \\ 'Department of Neurology, \\ The Second Affiliated Hospital of \\ Nanchang University, Nanchang, \\ People's Republic of China; \\ ${ }^{2}$ Department of Neurology, Affiliated \\ Hospital of Jiujiang University, \\ Jiujiang, People's Republic of China; \\ ${ }^{3}$ Department of Neurology, The Sixth \\ Hospital of Wuhan, Wuhan, People's \\ Republic of China
}

Background: It has been found that nuclear factor erythroid 2-related factor 2/antioxidant response element (Nrf2-ARE) signaling pathway plays a role in antioxidative response, antiinflammatory response, and neuron-protection in intracerebral hemorrhage (ICH). The aim of this study is to explore mechanisms underlying the perifocal neuroprotective effect of the Nrf2-ARE signaling pathway after ICH.

Methods: There were a total of 90 rats with basal ganglia hemorrhage, which were randomly divided into the following four groups: ICH (Sprague-Dawley rats with autologous femoral arterial blood injection into the basal ganglia), sulforaphane (SFN) (SFN was intraperitoneally administered into rats), retinoic acid (RA) (RA was intraperitoneally administered into rats), and dimethyl sulfoxide (the rats were treated with dimethyl sulfoxide). We observed the neurological score of the rats in the different groups, and collected brain tissues for immunofluorescence, Western blot, and reverse transcription polymerase chain reaction to detect expression of Nrf2, heme oxygenase (HO-1), nuclear factor- $\kappa \mathrm{B}(\mathrm{NF}-\kappa \mathrm{B})$, and tumor necrosis factor- $\alpha$ (TNF- $\alpha$ ).

Results: The results indicated that neurological dysfunction of rats was significantly improved in the SFN group, and the expressions of Nrf2 and HO-1 in tissues surrounding the hemorrhage were increased. Also, the level of NF- $\kappa \mathrm{B}$ and TNF- $\alpha$ were reduced compared to the ICH group. The RA group exhibited more severe neurological dysfunction and lower levels of Nrf2 and HO-1 than the SFN and ICH groups. Compared to the ICH group, the NF- $\kappa$ B and TNF- $\alpha$ expression in the RA groups was increased. In conclusion, RA inhibits Nrf2 dissociation and translocation into nucleus, thereby suppressing the anti-inflammatory effect of Nrf2-ARE signaling pathway. The activation of Nrf2-ARE signaling pathway by SFN can elevate expression of antioxidant enzyme HO-1, reduce perifocal inflammatory response after $\mathrm{ICH}$, and thus may play a neuroprotective role.

Conclusion: The results suggest that Nrf2-ARE signaling pathway may serve as a new target for treatment of perifocal inflammatory injury caused by ICH.

Keywords: cerebral hemorrhage, injuries, NF-E2-related factor 2, antioxidant response elements

\section{Introduction}

The incidence, morbidity, and mortality of intracerebral hemorrhage (ICH) remain high, yet the existing medical and surgical treatment methods are unable to prevent perifocal damage. The activation of inflammatory cells and the release of inflammatory cytokines in the perifocal tissues after ICH are key factors in triggering secondary brain damage. ${ }^{1,2}$ As an important regulator of the inflammatory response, nuclear transcription factor- $\mathrm{\kappa B}(\mathrm{NF}-\mathrm{\kappa B})$ can trigger and amplify the inflammatory response, and inhibition of the NF- $\kappa \mathrm{B}$ inflammatory response after ICH may alleviate neuronal damage. In addition, nuclear factor erythroid 2-related factor 2 (Nrf2) is an important 
regulator in the antioxidative response of the cells, it interacts with antioxidant response element (ARE), and plays a wide range of cytoprotective roles in antioxidative, antiapoptotic, and anti-inflammatory responses and neuroprotection. ${ }^{3,4}$

Studies by our group and others have found that the Nrf2 activator sulforaphane (SFN) can protect neurons by inducing the expression of heme oxygenase (HO-1) and quinone oxidoreductase 1 to protect neurons. ${ }^{5} \mathrm{HO}-1$ can serve as an important antioxidant enzyme and antagonize oxidative stress caused by brain damage. Therefore, the $N r f 2$ gene may become a new target for treatment of perifocal damage after ICH. In a previous study, Nrf2 knockout mice and normal mice were compared to explore the relevant protein expressions in the Nrf2-ARE signaling pathway and its role in $\mathrm{ICH}$, and the results suggested that compared to normal mice, the Nrf2 knockout mice showed more severe neurological deficits after $\mathrm{ICH}$, and the protein expression downstream of the pathway was very low. ${ }^{6}$ To investigate the mechanisms of Nrf2-ARE pathway further, we used SFN to activate the Nrf2-ARE signaling pathway, and retinoic acid (RA) was used to inhibit the Nrf2-ARE signaling pathway to examine the neuroprotective effect of HO-1 on perifocal tissues after $\mathrm{ICH}$ and to explore the underlying mechanisms. The goal was to control perifocal damage caused by $\mathrm{ICH}$ from its underlying molecular mechanisms, and to save neurons to the maximal possible degree. ${ }^{7}$

\section{Materials and methods Experimental animals}

In the present study, male Sprague Dawley rats (purchased from Hunan Slack Jiangda Laboratory Animal Co., Ltd. (Changsha, Hunan province, People's Republic of China); certificate number: SCXK [Xiang] 2009-0004) were used, and their body weights ranged from 250 to $300 \mathrm{~g}$. All animal handling procedures were performed according to the Guide for the Care and Use of Laboratory Animals of the National Institutes of Health and followed the guidelines of the Animal Welfare Act. All animal experiments were approved by the Experimental Animal Ethical Committee of Affiliated Hospital of Jiujiang University.

\section{Main reagents and instrument}

RA (specification: $1 \mathrm{~g}$ ), SFN (specification: $5 \mathrm{mg}$ ), and dimethyl sulfoxide (DMSO) (specification: $100 \mathrm{~mL} /$ bottle) were purchased from Sigma-Aldrich Co. (St Louis, MO, USA). The following primary antibodies were used: rabbit anti-rat Nrf2 polyclonal antibody and goat anti-rat HO-1 polyclonal antibody purchased from Abcam (Cambridge, UK); and rabbit anti-rat NF- $\kappa$ B polyclonal antibody and rabbit anti-rat tumor necrosis factor (TNF- $\alpha$ ) polyclonal antibody purchased from Cell Signaling Technology (Beverly, MA, USA). The following secondary antibodies were used: dog anti-rabbit polyclonal antibody labeled with Cy3 fluorescein and dog anti-goat polyclonal antibody labeled with fluorescein isothiocyanate, which were purchased from Abcam; and rabbit anti-rat $\beta$-actin polyclonal antibody, which was purchased from Abcam. The sodium dodecyl sulfate-polyacrylamide gel electrophoresis (SDS-PAGE) gel preparation kit was purchased from Beyotime Institute of Biotechnology (Shanghai, People's Republic of China). Instruments included the following: animal skull stereotaxic apparatus, electronic analytical balance, image analyzer, and ultraviolet spectrophotometer.

\section{Establishment of rat $\mathrm{ICH}$ model and magnetic resonance imaging verification}

The rats were subjected to fasting without water deprivation 8 hours before surgery. The method reported by Deinsberger et $\mathrm{al}^{7}$ was used for slow blood injection on stereotax at $3 \mathrm{~mm}$ lateral to the right side of the anterior fontanelle and a penetration depth of $5 \mathrm{~mm}$. Forty microliter autologous blood was slowly injected into the basal ganglia to establish a rat ICH model. For each rat, blood injection took approximately 30 minutes. Garcia 18-point test ${ }^{8}$ was performed to assess the neurological function of the rats, and those with a score $\geq 3$ were considered usable subjects. In collaboration with the Radiology Department of Tongji Hospital, affiliated with Huazhong University of Science and Technology and Tongji Medical College, the GE signa Excite HD 3.0 T MR scanner was used to examine the hematoma 3 hours after the ICH had been established (Figure 1). The scan sequence and details were listed as follows: coronal section T2WI (weighted image), $\mathrm{T} 2 * \mathrm{WI}$, thickness $0.8 \mathrm{~mm}$, interval $0.1 \mathrm{~mm}$. If a notable round, oval, or irregularly-shaped hematoma was observed, the model was considered to be successfully established. If there was blood reflux into the needle tract, blood in the ventricles, or death, the animal was excluded from the study.

\section{Blood pressure monitor}

The pentobarbital sodium was injected intraperitoneally, and the blood pressure was monitored during the experiments. The blood pressure was monitored by using tail venous manometry during the experiments. The monitor indicated that the blood pressure was stable during the whole of the experiments.

\section{Drug dosage and route of administration}

Five milligrams SFN was dissolved in $5 \mathrm{~mL}$ DMSO and intraperitoneally administered postoperatively into the rats at a concentration of $1 \mathrm{mg} / \mathrm{kg}$ twice daily. 

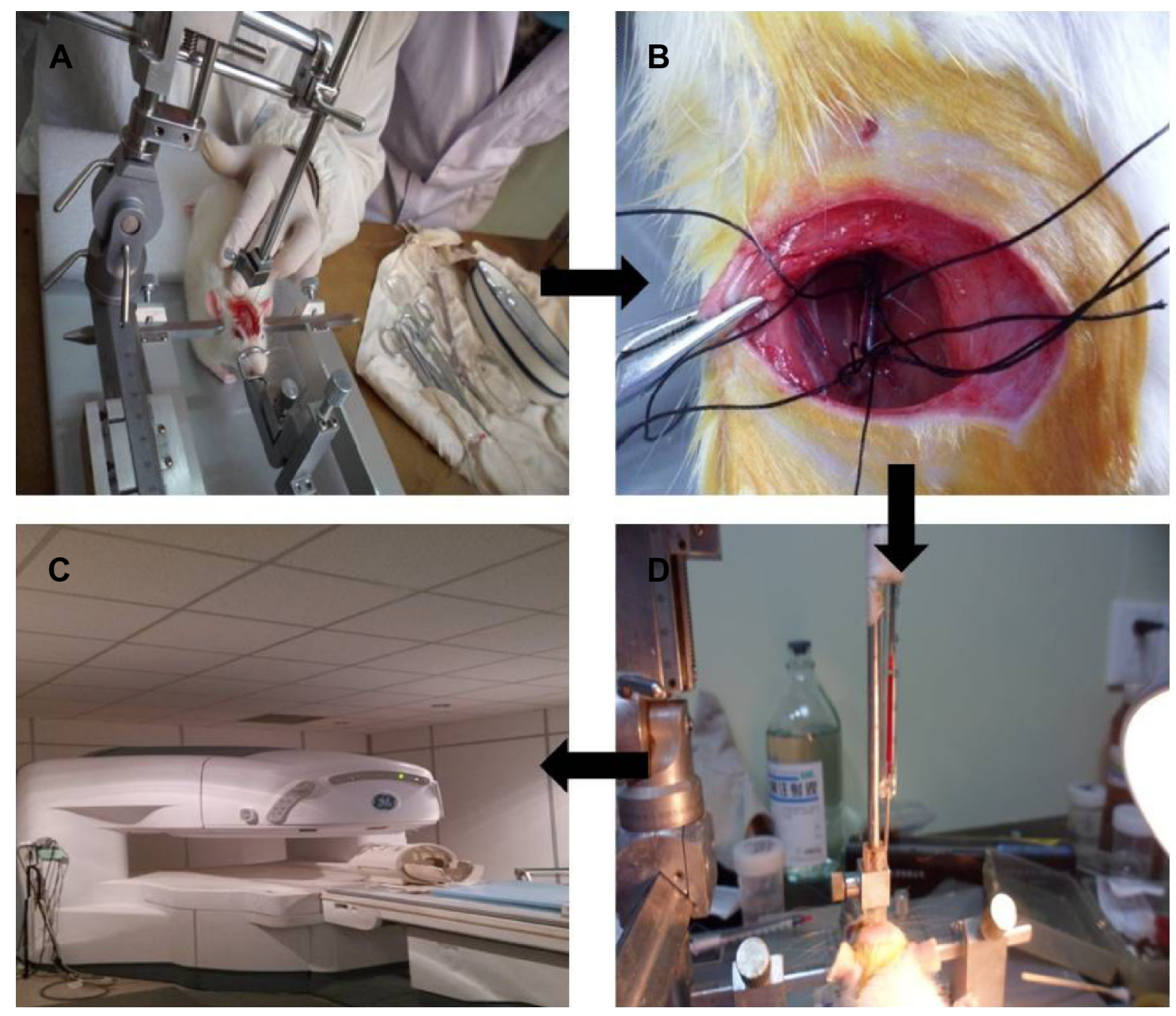

Figure I Establishment processes of ICH model.

Notes: (A) Positioning of the rat basal ganglia using the three-dimensional stereotax. (B) into the basal ganglion. (D) Rat MRI scan.

Abbreviations: $\mathrm{ICH}$, intracerebral hemorrhage; $\mathrm{MRI}$, magnetic resonance imaging.

One gram RA was dissolved in DMSO to a final concentration of $20 \mathrm{mg} / \mathrm{mL}$ and intraperitoneally administered 30 minutes after the surgery at a dose of $10 \mathrm{mg} / \mathrm{kg}$.

\section{Experimental groups and sample collection}

One-hundred healthy male Sprague-Dawley rats, weighing $250-300 \mathrm{~g}$, were used in the experiments. There were a total of 90 rats with basal ganglia hemorrhage, which were randomly divided into the following four groups: ICH, SFN, RA, and DMSO group. Each group was divided into the following five subgroups: 6 hours, 1 day, 2 days, 3 days, and 7 days group (after ICH), and each subgroup contained five rats.

The rats were anesthetized and subjected to a thoracotomy at the corresponding time points. The heart was rapidly exposed, and a perfusion tube was inserted from the left ventricle to the root of the aorta. A small cut was made on the right atrial appendage with scissors. Then, $200 \mathrm{~mL} 4^{\circ} \mathrm{C}$ saline was infused until the effluent fluid was clear, and 4\% paraformaldehyde was used for perfusion until the limbs of the rat were rigid. The animal was then decapitated and its brain collected, placed in $4 \%$ paraformaldehyde for 24 hours, and subsequently subjected to frozen sectioning at a slice thickness of $5 \mu \mathrm{m}$ for immunofluorescence staining.

\section{Neurological score}

The Garcia 18-point method was used to evaluate the neurological function of rats after ICH. The scores were obtained 24 hours before surgery, immediately before surgery, and 6 hours, 1 day, 2 days, 3 days, and 7 days after surgery.

\section{Immunofluorescence tests for detecting the expressions of Nrf2, $\mathrm{HO}-\mathrm{I}, \mathrm{NF}-\mathrm{KB}$, and TNF- $\alpha$}

Nrf2-, HO-1-, NF-кB-, and TNF- $\alpha$-positive cells were detected as follows: 1) frozen $5 \mu \mathrm{m}$ thick sections of brain tissue were 
fixed in precooling paraformaldehyde for 20-30 minutes; 2) phosphate-buffered saline (PBS) rinse was performed for 5 minutes three times, followed by membrane rupture using $0.25 \%$ triton for 15 minutes; 3) PBS rinse was performed for 5 minutes, three times, followed by blocking using $10 \%$ Bull serum albumin (BSA) for 60 minutes; 4) PBS rinse was performed for 5 minutes, three times, followed byincubation with primary antibody at $4^{\circ} \mathrm{C}$ overnight (approximately 12 hours); 5) PBS rinse was performed for 10 minutes, three times, followed byincubation with second antibody at room temperature for 2 hours, and all the next steps were performed in darkness; 6) PBS rinse was performed for 10 minutes, three times, followed by 4',6-diamidino-2-phenylindole staining for 15 minutes; 7) PBS rinse was performed for 5 minutes, three times, followed by mounting with glycerol, and fluorescent microscope was used for observation and taking images; and 8) OPTPro imaging analysis system (LI-COR Biosciences, Lincoln, NE, USA) was used to scan and analyze the images. At $200 \times$ magnification, images from five nonoverlapping fields were analyzed for cell counts and the mean was calculated.

\section{Western blot analysis for determining the protein expression levels of $\mathrm{Nrf2}, \mathrm{HO}-\mathrm{I}$, NF- $\kappa B$, and TNF- $\alpha$}

The brain tissues were removed from the $-80^{\circ} \mathrm{C}$ freezer and slowly thawed at room temperature. Tissues within $4 \mathrm{~mm}$ of the hematoma were collected and a radioimmunoprecipitation assay lysis buffer containing a protease inhibitor cocktail (1:9, ie, $100 \mathrm{mg}$ brain tissue added into $0.9 \mathrm{~mL}$ buffer) was added before the tissue was subjected to homogenization. All the above steps were performed on ice. After homogenization, the mixture was placed on ice for another 30 minutes to allow full cleavage of the proteins. Next, centrifugation at $12,000 \times \mathrm{g}$ for 30 minutes at $4^{\circ} \mathrm{C}$ was performed and the supernatant was collected. The bicinchoninic acid (BCA) assay was performed to measure protein concentration (BCA kit purchased from Beyotime Institute of Biotechnology), and based on the protein concentration, the sample volume in each well was calculated so that the total protein concentration in all wells was equal. After the loading buffer had been added, the protein was boiled before it was subjected to electrophoresis on 10\% SDS-PAGE gel. After completion of electrophoresis, wet transfer of the membrane onto a nitrocellulose membrane $(0.45 \mu \mathrm{m})$ was performed at $100 \mathrm{~V}$ constant voltage for 90 minutes. Then, the membrane was blocked in 5\% skim milk at room temperature for 1 hour. After blocking, anti-Nrf2 (1:1,000, ab31163; Abcam), anti-HO-1 (1:2,000, ab13243; Abcam), anti-NF-kB (1:1,000, CST\#8242; Cell Signaling Technology), and anti- $\beta$-actin (1:1,000, 70-Mab1445; Sigma-Aldrich Co.) were added and the membranes were incubated at $4^{\circ} \mathrm{C}$ overnight. On the next day, $1 \times$ PBS was used to rinse the membranes for 10 minutes, three times, before they were incubated with Odyssey fluorescent secondary antibodies $(1: 15,000)$ in the dark for 1 hour. Subsequently, $1 \times$ PBS was used to rinse for 10 minutes, three times. The Tow-color Laser Odyssey Infrared Imaging System (Gene Company Limited, Hong Kong, People's Republic of China) was used to scan the membranes, and the images were analyzed using Odyssey Version 3.0 software. The ratio of the grayscale value of the target protein to that of $\beta$-actin was used for statistical analysis.

\section{Reverse transcription polymerase chain reaction detection of the $m R N A$ levels of Nrf2, HO-I, NF- $\kappa B$, and TNF- $\alpha$}

Real-time fluorescent quantitative polymerase chain reaction (PCR) was performed to examine the expressions of $\mathrm{Nrf2}$, HO-1, NF- $\kappa B$, and TNF- $\alpha$. All primers were synthesized by Beijing Qing Ke New Industrial Biotechnology Co., Ltd. (Beijing, People's Republic of China), and the primers sequences are shown in Table 1. Reverse transcription PCR (RT-PCR) was performed as follows. The rat was sacrificed at the corresponding time point, and approximately $20 \mathrm{mg}$ brain tissue was rapidly collected on a super-clean bench. TRIzol reagent (Takara Co., Ltd., Dalian City, Liaoning Province, People's Republic of China) was used for single-step extraction of total RNA, and the RNA quality and concentration were tested at optical density 260/280. Then, $5 \mu \mathrm{g}$ total RNA was used for reverse transcription reaction with the Real-Time PCR System (Thermo Fisher Scientific, Waltham, MA, USA).

Table I The primers of the genes

\begin{tabular}{llll}
\hline Target gene & Forward primer $\mathbf{( 5}$ ' to $\mathbf{3}^{\prime}$ ) & Reverse primer (5' to $\mathbf{3}^{\prime}$ ) & Size (bp) \\
\hline Nrf2 & GAATAAAGTTGCCGCTCAGAA & AAGGTTTCCCATCCTCATCAC & 209 \\
HO-I & CTATCGTGCTCGCATGAAC & CAGCTCCTCAAACAGCTCAA & II8 \\
NF-KB & ACGATCTGTTTCCCCTCATCT & TGCTTCTCTCCCCAGGAATA & 150 \\
TNF- $\alpha$ & GACCCTCACACTCAGATCATC & GAACCTGGGAGTAGATAAGG & 197 \\
GAPDH & ACTCCCATTCCTCCACCTTT & TTACTCCTTGGAGGCCATGT & I43 \\
\hline
\end{tabular}


After reverse transcription, all cDNA was diluted to $150 \mathrm{ng} / \mu \mathrm{L}$, and $2 \mu \mathrm{L}$ cDNA (diluted) was used for PCR (reagents purchased from Takara Co., Ltd., Dalian City, Liaoning Province, People's Republic of China [\#RR820A]). The cycle threshold was recorded. The results of the real-time quantitative PCR were automatically provided by the quantitative fluorescence analyzer, including the cycle threshold values of the target genes and the reference gene, as well as $-\Delta \Delta \mathrm{CT}$. Experiments on each group of samples were repeated three times.

\section{Statistical analysis}

SPSS 19.0 statistics software was used for data analysis. Data are expressed as means \pm standard deviation. For comparison of multiple groups, univariate analysis of variance was performed. For comparison of two groups, the $t$-test was performed. $P<0.05$ was considered statistically significant.

\section{Results \\ Magnetic resonance imaging scans of hematomas in rats after $\mathrm{ICH}$}

In collaboration with the Radiology Department of Tongji Hospital, affiliated with Huazhong University of Science and Technology and Tongji Medical College, the GE signa Excite
HD 3.0 T MR scanner was used to examine the hematomas 3 hours after ICH was established. The scan sequence was as follows: coronal section T2WI, T2*WI, slice thickness $0.8 \mathrm{~mm}$, and interval $0.1 \mathrm{~mm}$. Hematomas were observed in the right basal ganglion (Figure 2).

\section{Neurological score}

The neurological deficits of rats in the ICH group peaked on day 3 , and on day 7 there was still rather severe damage to the neurological function. On day 1 after surgery, the neurological score of the SFN group was significantly higher than that of the ICH group $(P<0.05)$. Compared to the RA group, the neurological function of rats in the SFN group was significantly improved $(P<0.05)$. The neurological deficits of rats in the RA group were notably more severe compared to the SFN and ICH groups (Figure 3). The data suggest that SFN intervention after ICH to activate the Nrf2-ARE pathway can significantly alleviate the neurological symptoms of the rats and improve the neurological score. RA inhibits the Nrf2-ARE pathway and aggravates secondary injury after ICH. The Garcia 18-point method was used to evaluate the neurological function of the rats, at 6 hours, 1 day, 2 days, 3 days, and 7 days after surgery.

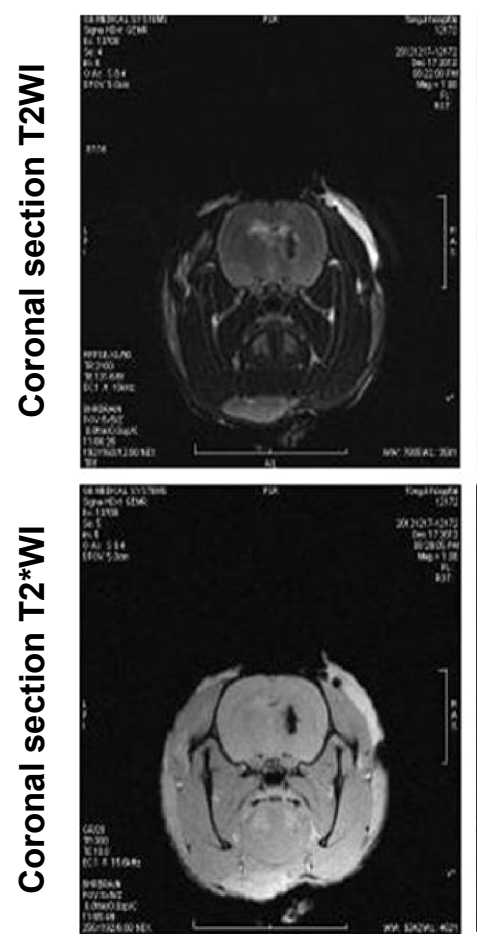

Rat 1
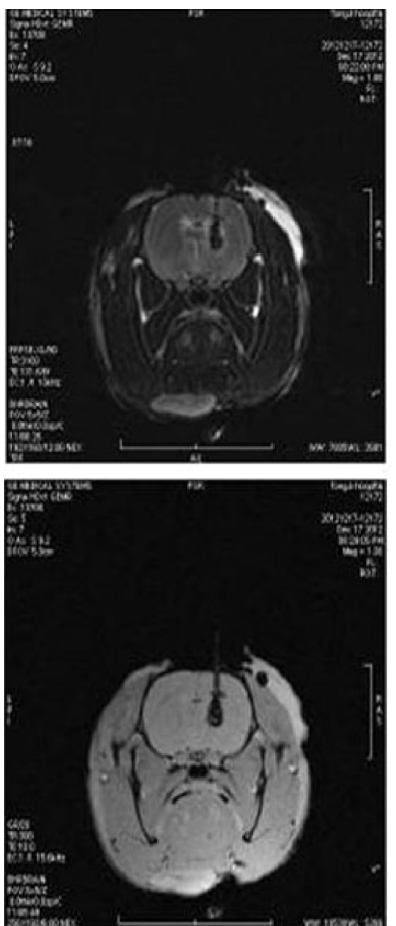

Rat 2
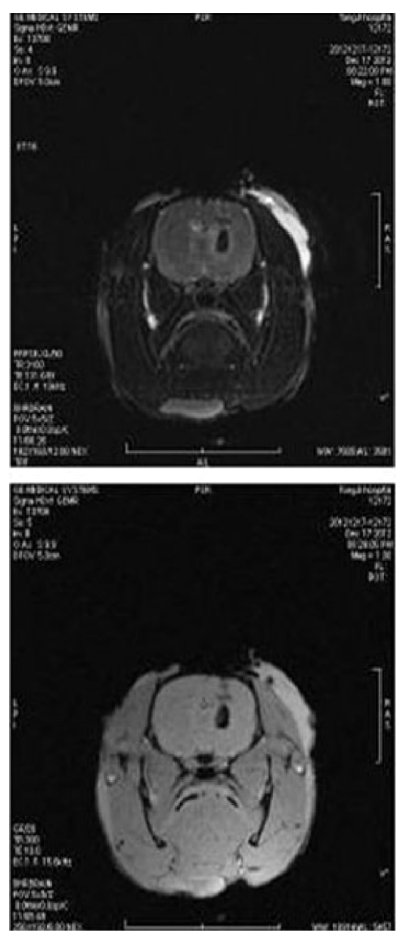

Rat 3

Figure 2 Coronal section T2Wl, T2*Wl, slice thickness $0.8 \mathrm{~mm}$, interval $0.1 \mathrm{~mm}$.

Notes: Hematoma in the right basal ganglion was detected. During MRI scan, the rat was in a prone position. For this figure, we selected the most standard MRI scan photos in one rat. The upper three images represent coronal section T2WI image, and the lower three images represent coronal section T2*WI image.

Abbreviations: MRI, magnetic resonance imaging; WI, weighted image. 


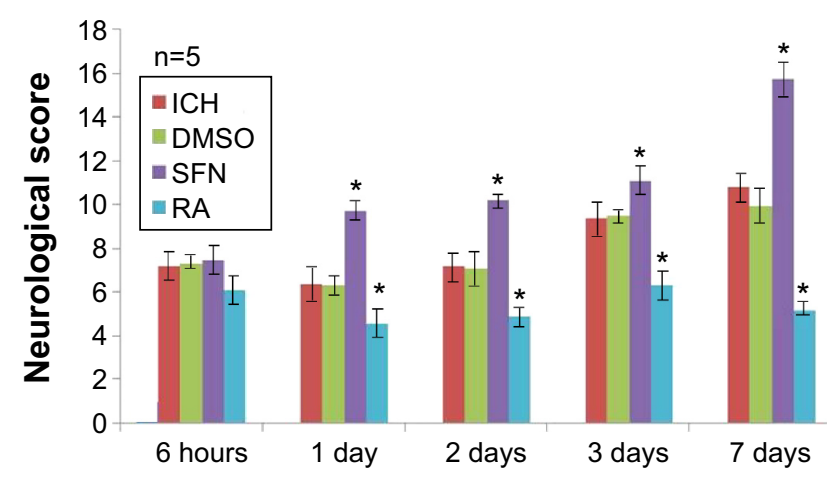

Figure 3 With time, the neurological dysfunction in rats of the SFN group improved. Note: $* P<0.05$ represents the neurological scores in the SFN group or RA group compared to the $\mathrm{ICH}$ group.

Abbreviations: DMSO, dimethyl sulfoxide; $\mathrm{ICH}$, intracerebral hemorrhage; RA, retinoic acid; SFN, sulforaphane.

\section{Coexpression of $\mathrm{Nrf2}$ and $\mathrm{HO}-\mathrm{I}$ detected by double immunofluorescence staining}

To further investigate the synergistic effect of $\mathrm{HO}-1$ downstream of the Nrf2-ARE signaling pathway on ICH perifocal tissues, coexpression of Nrf2 and HO-1 was examined by double immunofluorescence staining. The 3-day and 7-day subgroups in the SFN group were compared. We found that the cells positive for coexpression of $\mathrm{Nrf} 2$ and $\mathrm{HO}-1$ reached a peak on day 3, which suggests that HO-1 may be an important target in neuron protection. The expression of $\mathrm{Nrf} 2$ and the downstream target protein HO-1 in the RA group was significantly decreased compared to both the ICH and SFN groups (Figures 4-6).
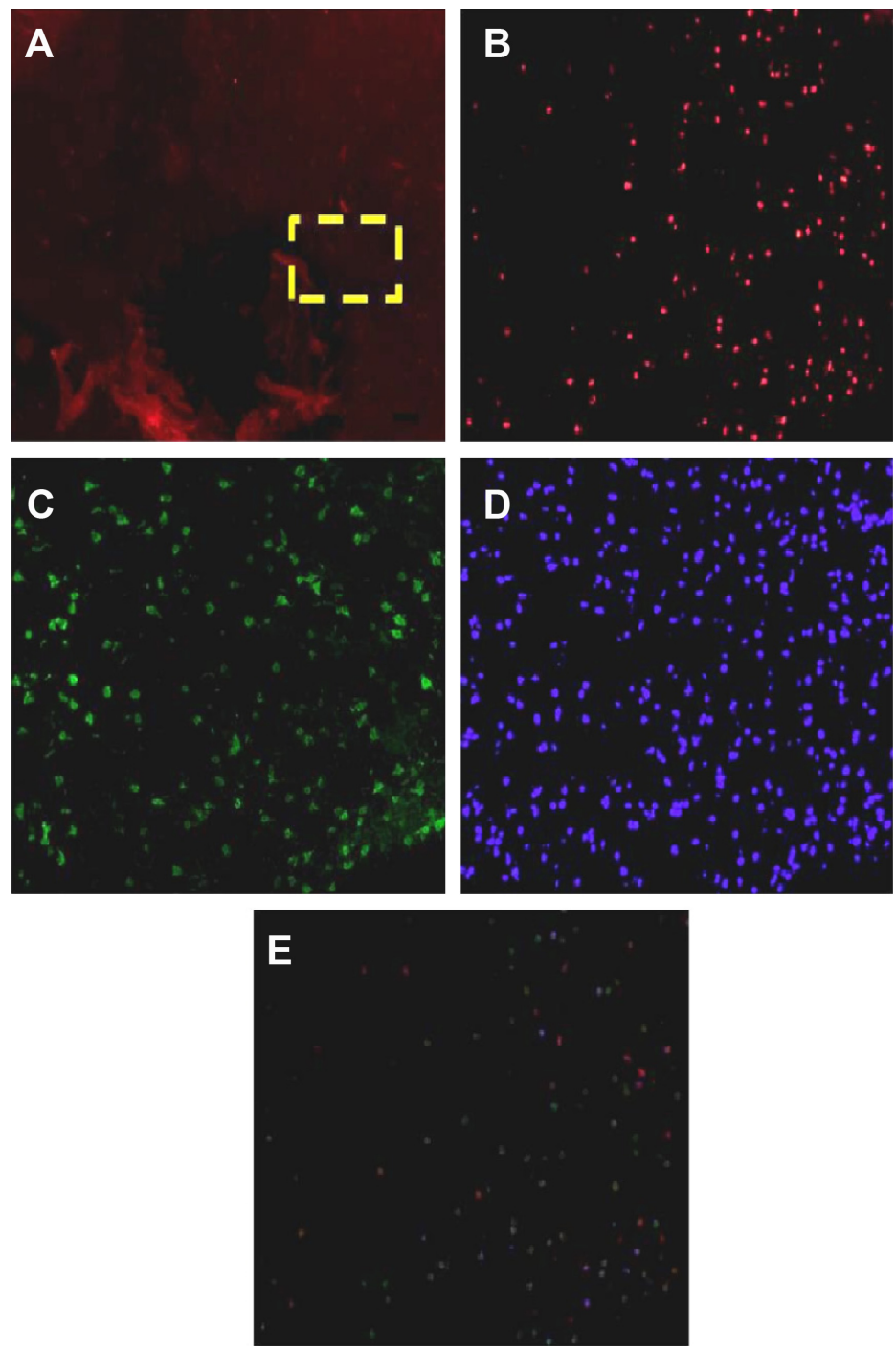

Figure $4 \mathrm{Nrf2}$ protein and $\mathrm{HO}-\mathrm{I}$ protein expression under the immunofluorescence.

Notes: (A) Expression of target proteins in perifocal tissues within $4 \mathrm{~mm}$ from the focus of the ICH, as revealed by immunofluorescence staining under low magnification $(40 \times)$. The yellow box is the target area where the protein expression around the cerebral hemorrhage was observed. (B) Nrf2-positive cells revealed at high magnification (200x). (C) HO-I-positive cells revealed at high magnification (200×). (D) Nuclear staining by 4',6-diamidino-2-phenylindole (200× magnification). (E) Integration and merged image of panels $(\mathbf{A}-\mathbf{C})$ and cell counting using software.

Abbreviations: Nrf2, nuclear factor erythroid 2-related factor 2; ICH, intracerebral hemorrhage; $\mathrm{HO}$ - I, heme oxygenase. 
A
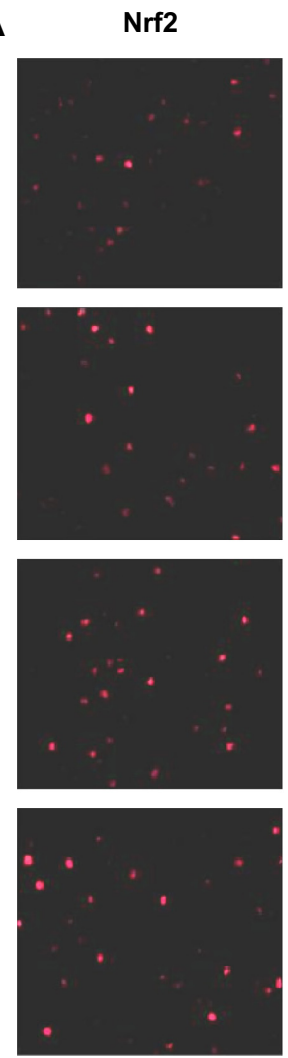

B
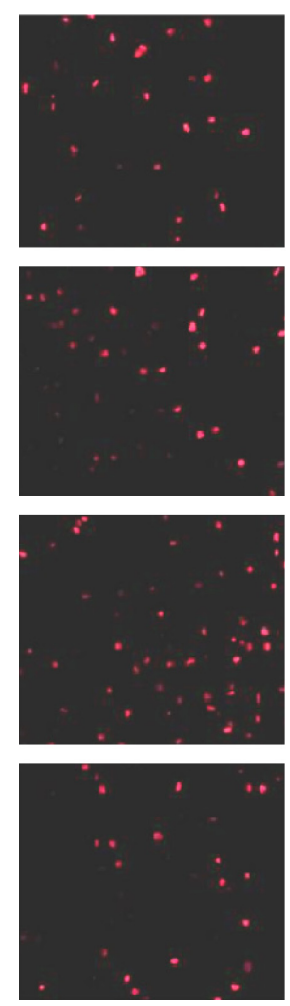

HO-1
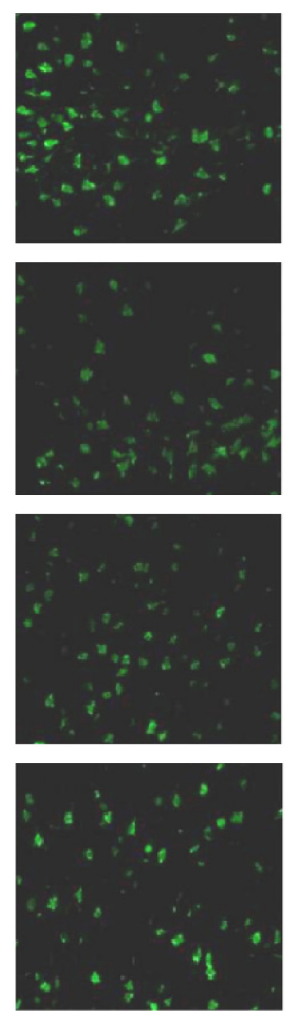

HO-1
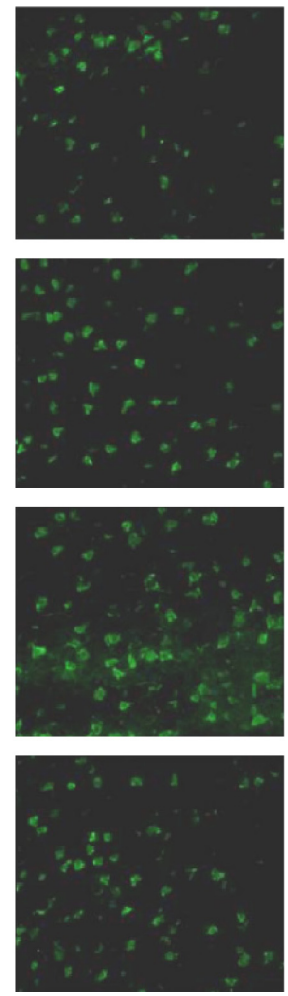

DAPI
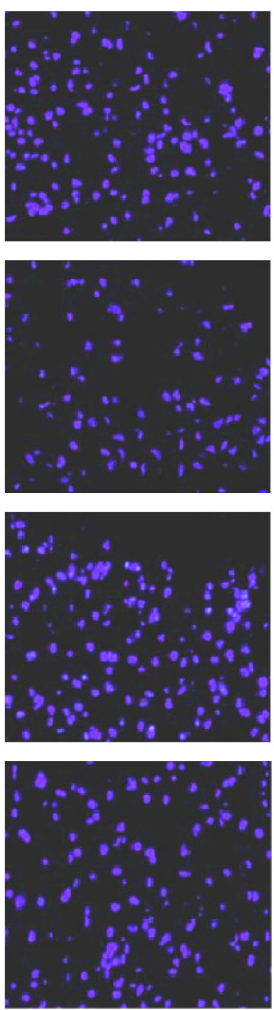

DAPI
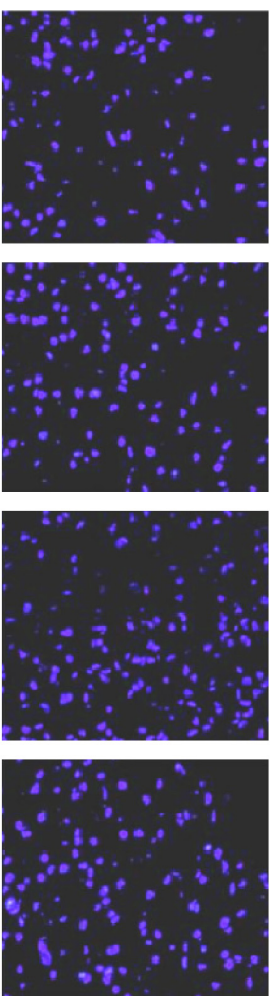

Merge

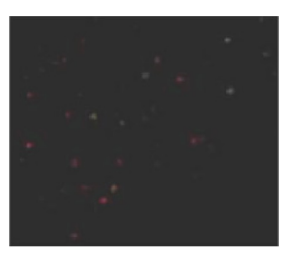

ICH 3 days
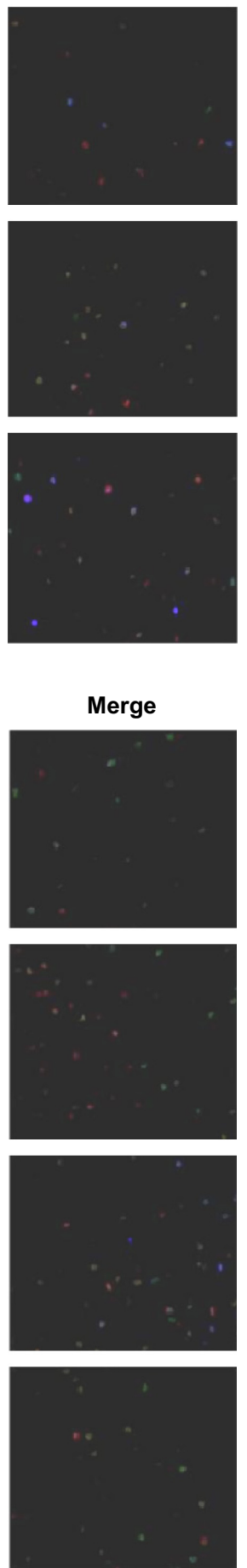

RA 3 days

ICH 7 days

DMSO 7 days

DMSO 3 days

SFN 3 days

SFN 7 days

RA 7 days

Figure 5 Cells stained positive for both $\mathrm{Nrf2}$ and $\mathrm{HO}-\mathrm{I}$ protein.

Notes: (A) Cells stained positive for both Nrf2 and HO-I at 3 days (400× magnification). (B) Cells labeled positive for both Nrf2 and $\mathrm{HO}-\mathrm{I}$ at 7 days (400 $\times$ magnification). In the SFN group, on day 7 the number of cells double-stained for Nrf2 and HO-I was significantly increased compared to day 3 (P<0.0I). In addition, compared to the RA group, the ICH and DMSO groups had a significantly higher number of cells double-stained for Nrf2 and $\mathrm{HO}-\mathrm{I}(P<0.0 \mathrm{I})$.

Abbreviations: DAPI, 2-(4-Amidinophenyl)-6-indolecarbamidine dihydrochloride; DMSO, dimethyl sulfoxide; HO-I, heme oxygenase; Nrf2, nuclear factor erythroid 2-related factor 2; ICH, intracerebral hemorrhage; RA, retinoic acid; SFN, sulforaphane. 
A

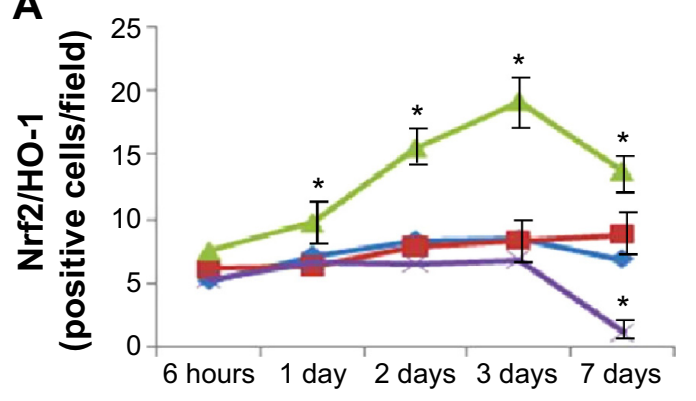

B

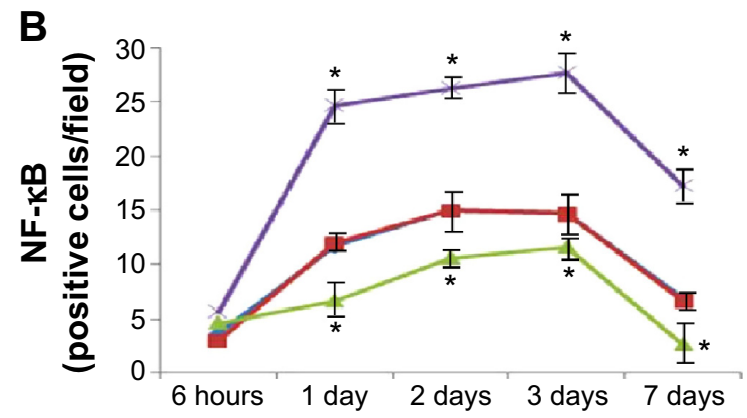

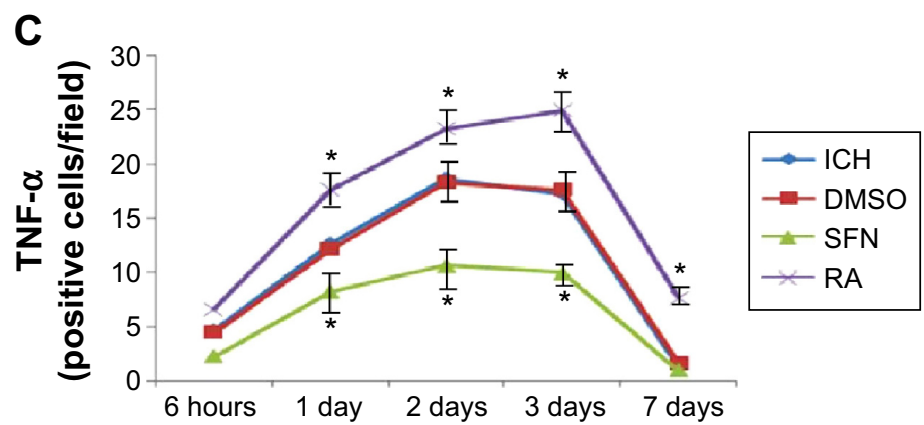

Figure 6 Cells double-stained for Nrf2, HO-I, NF- $\kappa$ B, and TNF- $\alpha$.

Notes: (A) Cells doubled-stained for Nrf2 and HO-I. On day 3 the number of the double-stained positive cells in the SFN group was the largest; the number of the doublestained positive cells in the RA group was significant smaller than in the SFN group $(P<0.05)$. ( $\mathbf{B}$ and $\mathbf{C})$ Compared to both the RA and the ICH groups, the expression of NF- $K B$ and TNF- $\alpha$ in the SFN group was significantly reduced $(P<0.05)$ suggesting the expression of anti-inflammatory substances after activation of Nrf2 and HO-I. The expressions of various proteins in the $\mathrm{ICH}$ group as shown in panels $(\mathbf{A}-\mathbf{C})$ were not different from that in the DMSO group, suggesting that as a solvent, DMSO does not have any confounding effects. ${ }^{*} \mathrm{P}<0.05$ represents the indexes in the SFN group or RA group compared to the ICH group.

Abbreviations: DMSO, dimethyl sulfoxide; ICH, intracerebral hemorrhage; HO-I, heme oxygenase; Nrf2, nuclear factor erythroid 2-related factor 2; TNF $\alpha$, tumor necrosis factor- $\alpha$; NF- $\kappa B$, nuclear factor- $\kappa B$; RA, retinoic acid; SFN, sulforaphane.

Also, the Nrf2 could coexpress with NF-kB and TNF- $\alpha$ protein. The results indicated that the expression of NF- $\mathrm{\kappa B}$ and the Nrf2 protein in the RA group was significantly increased compared to both the ICH and SFN group (Figure S1).

\section{Nrf2, HO-I, NF- $\kappa B$, and TNF- $\alpha$ protein expressions revealed by Western blot analysis}

Compared to the ICH group, the expressions of Nrf2 and HO-1 proteins in the DMSO group were not significantly different $(P>0.05)$. Compared to both the SFN and ICH groups, the Nrf2 protein expression in the RA group at 2 days, 3 days, and 7 days (Figure 7) was substantially reduced. After correction for drift by internal reference, we found that these differences were statistically significant.

\section{mRNA levels of Nrf2, HO-I, NF- $\mathrm{HB}$, and TNF- $\alpha$ revealed by RT-PCR}

RT-PCR results on tissues surrounding the focus of ICH showed that compared to the DMSO and ICH groups, the Nrf2 and HO-1 expressions in the SFN group were both significantly increased, whereas the NF- $\mathrm{BB}$ and TNF- $\alpha$ expression were both significantly decreased. Compared to the DMSO and ICH groups, the Nrf2 and HO-1 expression in the RA group were both significantly decreased, whereas the NF- $\mathrm{KB}$ and TNF- $\alpha$ expression were both significantly increased (Figure 8).

\section{Discussion}

Activation of the Nrf2-ARE signaling pathway can induce the expression of endogenous protective genes in a variety of tissues and cells, including antioxidant enzymes, anti-inflammatory factors, and many detoxifying proteins. When these antioxidants and anti-inflammatory factors increase their expression in neurons and glial cells, these cells are protected against the oxidative damages induced by dopamine, glutamate, and hydrogen peroxide. ${ }^{9,10}$ Activation of inflammatory cells and release of inflammatory cytokines in perifocal tissues after ICH may be crucial to secondary brain damage. The results of the present study showed that RA can inhibit the translocation of Nrf2 into the nucleus, thereby inhibiting the anti-inflammatory effect of the Nrf2-ARE signaling pathway, whereas activation of the Nrf2-ARE signaling pathway by SFN can elevate the expression of the antioxidant enzyme HO-1, thereby reducing the perifocal inflammatory response after ICH and enhancing neuroprotection. 
A

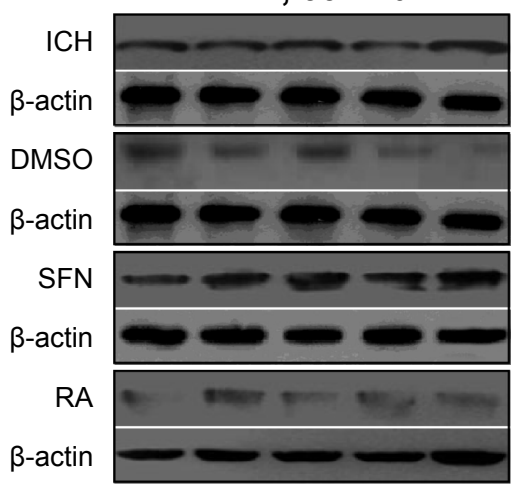

B

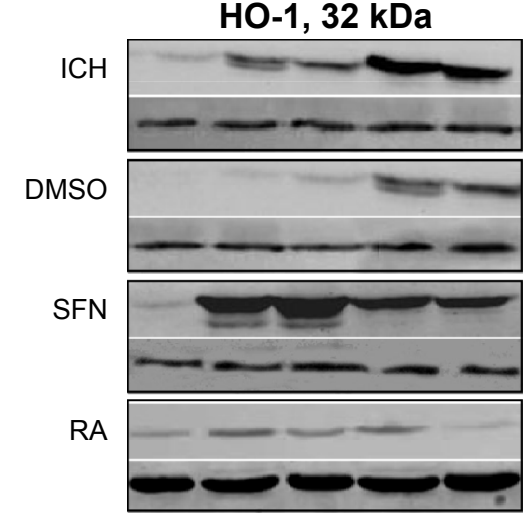

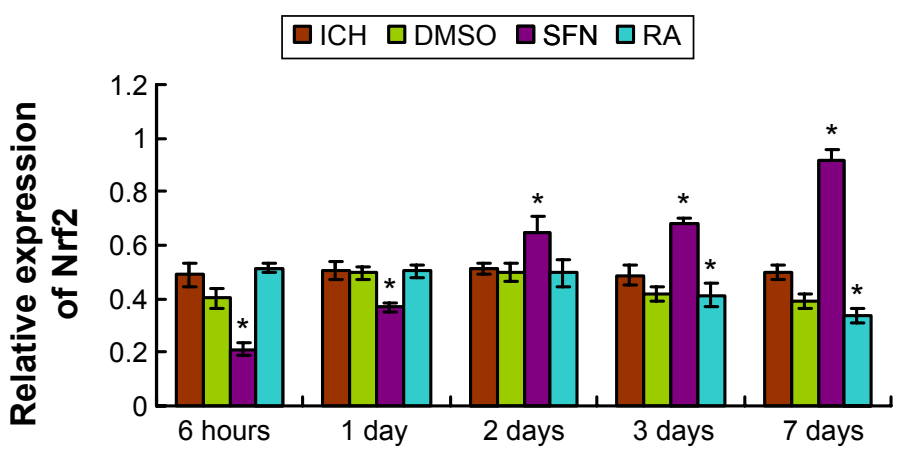

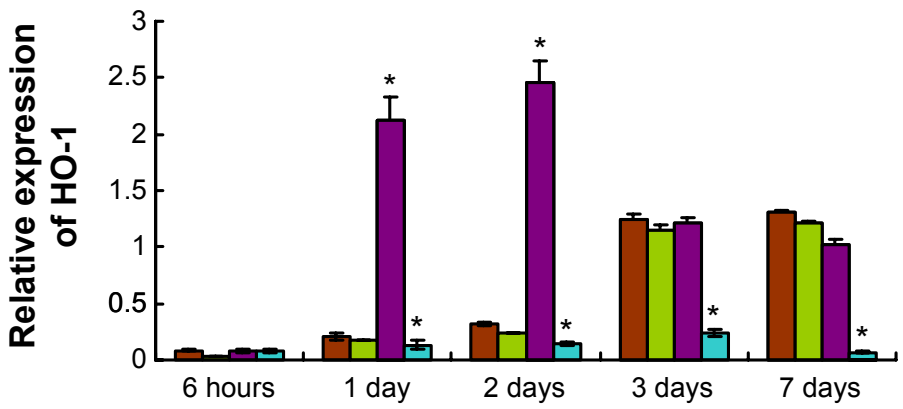

Figure 7 Western blot assay of the Nrf2 and HO-I protein.

Notes: (A) Compared to the ICH group, the total protein levels of $\mathrm{Nrf2}$ in the SFN group at all time points were significantly elevated $(P<0.05)$; compared to the SFN group and the $\mathrm{ICH}$ group, the total protein levels of $\mathrm{Nrf2}$ at I day, 2 days, 3 days, and 7 days in the RA group were notably decreased; after correction of drift by internal reference, these differences were found to be significant $(P<0.01)$. (B) Western blot analysis revealed that compared to both the ICH and the RA groups, the total protein levels of $\mathrm{HO}-\mathrm{I}$ at all time points in the SFN group were elevated, and the differences between the SFN and the RA groups for all time points were statistically significant $(P<0.05)$. These results suggest that activation of $\mathrm{Nrf2}$ after $\mathrm{ICH}$ can upregulate the expression of HO- I. *statistically significant.

Abbreviations: DMSO, dimethyl sulfoxide; ICH, intracerebral hemorrhage; HO-I, heme oxygenase; Nrf2, nuclear factor erythroid 2-related factor 2; RA, retinoic acid; SFN, sulforaphane.

The Nrf2-ARE signaling pathway is a promising new target for the study of the treatment of ICH.

Studies have shown that Nrf2 is an important regulator in the antioxidative response of the cells. It interacts with ARE, and plays a wide range of cytoprotective roles in antioxidative, antiapoptotic, and anti-inflammatory responses and neuroprotection. ${ }^{11,12} \mathrm{Li}$ and $\mathrm{Nel}^{3}$ reported that the Nrf2 activator SFN could protect motor neurons by inducing the expressions of HO-1 and quinone oxidoreductase 1. Studies have shown that activation of Nrf2 by SFN can induce microglial activation, enhance phagocytosis of excess iron ions by microglia, and reduce free radicals, thereby playing a neuroprotective role. In addition, it can reduce the inflammatory response induced by lipopolysaccharide (LPS) and decrease the expression of inflammatory cytokines. ${ }^{1}$

The results of the present study showed that SFN activates the Nrf2-ARE signaling pathway, increases Nrf2 and HO-1 expression, and inhibits the expression of perifocal inflammatory substances NF- $\kappa \mathrm{B}$ and TNF- $\alpha$. Comparisons of neurological scores reveal that compared to the $\mathrm{ICH}$ group, the protein and mRNA expression of Nrf2 and HO-1 increase, whereas the expression of perifocal inflammatory substances NF- $\kappa \mathrm{B}$ and TNF- $\alpha$ decrease in the SFN group. This suggests that the Nrf2-ARE signaling pathway may be involved in the antioxidative and anti-inflammatory responses, inhibition of edema, and neuroprotection in perifocal tissues after ICH. To test our hypothesis further, RA intervention was provided after ICH model was established in the RA group. The results showed that the neurological deficits of the surviving rats were more severe. The data suggest that SFN can activate the Nrf2-ARE pathway and alleviate the neurological symptoms of the ICH rats, which is possibly caused by inhibiting brain edema and the expression of neurotoxic substances. In addition, the protein and mRNA expressions of Nrf2 and HO-1 were both reduced compared to the ICH and SFN groups. Western blot analysis revealed that there was more cytoplasm-bound Nrf2 in the RA than in the SFN group. This suggests that RA inhibits 
A

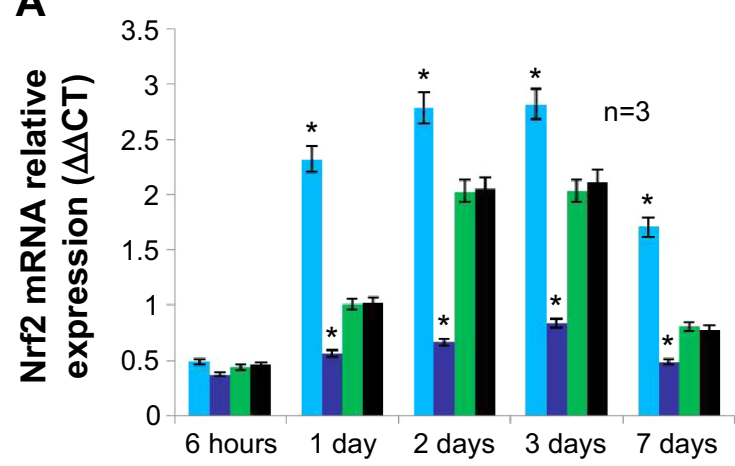

B

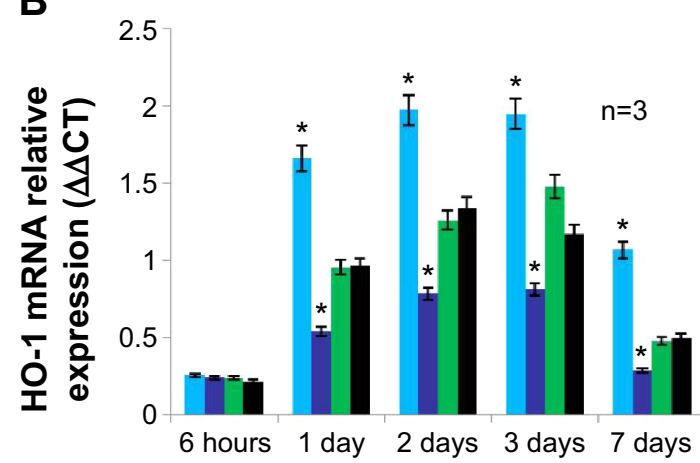

C

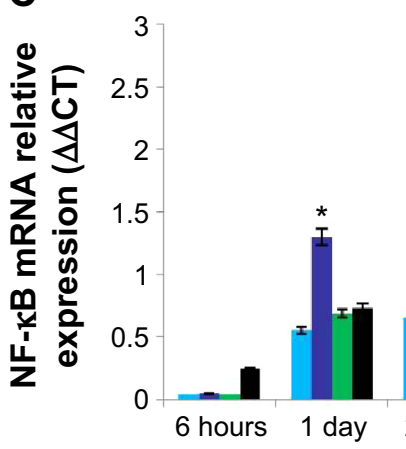

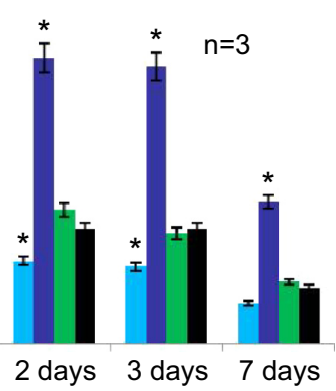

D

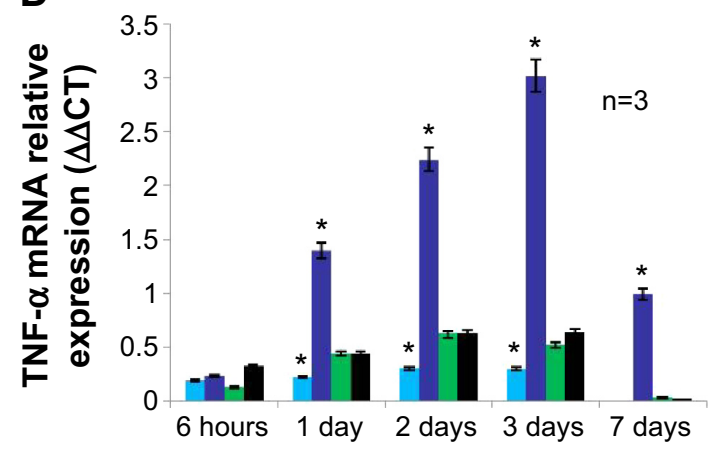

SFN $\square \mathrm{RA} \quad \mathrm{ICH} \square \mathrm{DMSO}$

Figure 8 The mRNA expression in Nrf2, HO-I, NF- $\kappa B$, and TNF- $\alpha$.

Notes: (A and B) show that compared to the RA and ICH groups, the Nrf2 and HO-I mRNA expression in the SFN group was significantly elevated $(P<0.05)$; this suggests that SFN induces the translocation of Nrf2 into the nucleus, and enhances the expression of anti-inflammatory protein $\mathrm{HO}$ - I downstream. (C and $\mathbf{D})$ show that compared to the RA and ICH groups, the expression of NF- $\mathrm{KB}$ and TNF- $\alpha$ in the SFN group was significantly decreased $(P<0.05)$; this suggests that after the activation of Nrf2 and HO-I mRNA expressions, anti-inflammatory proteins are expressed. The expressions of various substances in the ICH group in panels (A-D) were not different from that in the DMSO group, suggesting that as a solvent, DMSO does not have any confounding effects. *Statistically significant with SFN and RA.

Abbreviations: DMSO, dimethyl sulfoxide; ICH, intracerebral hemorrhage; HO-I, heme oxygenase; Nrf2, nuclear factor erythroid 2-related factor 2; NF- $\mathrm{KB}$, nuclear factor$\kappa B$; TNF- $\alpha$, tumor necrosis factor- $\alpha$ RA, retinoic acid; SFN, sulforaphane.

the dissociation of Nrf2, reduces its translocation into the nucleus, and inhibits the expression of Nrf2 and HO-1. Meanwhile, the perifocal expression of NF- $\mathrm{KB}$ and TNF- $\alpha$ is elevated. ${ }^{13,14} \mathrm{We}$ demonstrated for the first time that as an Nrf2 inhibitor, the mechanism of the inhibitory effect of RA on the Nrf2-ARE signaling pathway may be through dissociation of Nrf2 and Keap1.

To explore the mechanisms, pathways, key proteins, and key cells underlying the inhibition of the NF-KB inflammatory signaling pathway after ICH by the Nrf2-ARE pathway, double immunofluorescent staining was performed to examine the coexpression of Nrf2 and HO-1 in perifocal tissues. The 1-day, 2-days, 3-days, and 7-days subgroups in the SFN group were compared, and the results revealed that coexpression of Nrf2 and HO-1 reached a peak on day 3 followed by sustained expression until day 7, indicating that HO-1 is located downstream of the Nrf2-ARE signaling pathway, which may be an important target protein in neuroprotection. RA is an Nrf2 inhibitor, the Nrf2 levels were significantly reduced in the RA group compared to both the ICH and SFN groups. HO-1 mediates neuroprotection possibly through the following mechanisms: 1) hemochrome can induce oxidative stress and inflammation, leading to cell damage, and HO-1 plays a detoxifying role by clearing the free hemochromes; and 2) hemochrome is highly lipophilic, upregulates the expression of cell and vascular adhesion molecules, and inhibits the NF- $\mathrm{KB}$ signal transduction pathway, thereby playing an anti-inflammatory role. Therefore, in future studies, we would use the specific HO-1 inhibitor zinc protoporphyrin (ZPP)+ to conduct relevant experiments to test the above hypotheses.

Other studies have not shown that activation of $\mathrm{Nrf} 2$ can increase the HO- 1 expression in microglia. HO- 1 can antagonize the effects of LPS-induced TNF- $\alpha$ and interleukin-1 (IL-1) by producing a low concentration of carbon monoxide, and it is a key anti-inflammatory factor that inhibits the macrophage migration factor. The underlying mechanism may be through inhibition of the NF- $\kappa$ B signaling pathway, 
inhibition of the dissociation between Inhibitor-KappaB

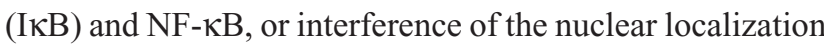
signal in the P65 subunit of NF- $\mathrm{KB} .{ }^{15,16}$

NF- $\kappa \mathrm{B}$ belongs to the Rel protein family. Typically, it binds to the I $\kappa \mathrm{B}$ protein and is localized in the cytoplasm in an inactive form. When cells are stimulated by inflammatory cytokines or chemical substances, NF- $\mathrm{KB}$ is dissociated from $\mathrm{I} \kappa \mathrm{B}$ and enters the nucleus, where it binds to the corresponding target sequence and regulates the transcriptional activity of relevant genes. A variety of factors can activate NF- $\kappa B$, such as TNF, IL-1, LPS, viral infection, and mitogenic factor. After NF- $\kappa \mathrm{B}$ translocates into the nucleus, it binds to NF- $\kappa \mathrm{B}$-binding sites and induces the transcription of the corresponding target genes. ${ }^{17-19}$ The results of the present study showed that NF- $\mathrm{KB}$ expression started within 6 hours after $\mathrm{ICH}$, followed by sustained cascade reactions, and in the ICH group, the high expression of NF- $\mathrm{\kappa B}$ persisted for 7 days, or even longer. This could produce serious damage to perifocal nerves. In the SFN group, activation of the Nrf2-ARE signaling pathway resulted in inhibition of NF- $\mathrm{KB}$ activity on day 3 . However, this inhibitory effect gradually declined later on. This result suggests that early activation of the Nrf2-ARE signaling pathway has a neuron-protective effect. These experiments on animal ICH models demonstrate that $\mathrm{NF}-\kappa \mathrm{B}$ is involved in the pathological process of perifocal injuries after ICH. Meanwhile, the etiology for the elevation of NF- $\kappa \mathrm{B}$ expression and its sustained high expression remains unclear. The microglial cells in the central nervous system, which are the safe guards in the system, have both neuroprotective and neurotoxic effects. It remains unclear what regulates the activation and function of microglia. These issues need to be addressed with further studies.

In summary, our results show that SFN activates the Nrf2-ARE signaling pathway to reduce the damage caused by oxidative stress and inflammation and the pathological progress of $\mathrm{ICH}$, and can effectively protect the brain tissues of ICH rats, thereby improving their neurological functions. RA inhibits the Keap1-Nrf2-ARE signaling pathway and enhances the expression of inflammatory factors. The mechanism underlying the neuron-protective effect of the Nrf2-ARE signaling pathway mainly involves inhibition of the inflammatory response and the neurotoxic effect of the NF- $\kappa \mathrm{B}$ pathway. It may also alleviate cerebral edema by improving the blood-brain barrier. The Nrf2-ARE signaling pathway may serve as a target for $\mathrm{ICH}$ treatment at the molecular level due to its involvement in antioxidative and anti-inflammatory responses, and its effect on other pathways.

\section{Conclusion}

In conclusion, activation of the Nrf2-ARE signaling pathway by SFN can alleviate the perifocal inflammatory response after $\mathrm{ICH}$, reduce neurological deficits, and increase the expression of the antioxidative enzyme $\mathrm{HO}-1$, and thereby may play a neuroprotective role. The Nrf2-ARE signaling pathway can serve as a new target for the study on $\mathrm{ICH}$ treatment.

\section{Acknowledgment}

Funding for the present study was granted by National Natural Science Foundation of China (No 81260183).

\section{Disclosure}

The authors report no conflicts of interest in this work.

\section{References}

1. Liu D, Dong Y, Liu Z, Niu B, Wang Y, Gao X. Impact of TREM-2 gene silencing on inflammatory response of endotoxin-induced acute lung injury in mice. Mol Cell Biochem. 2014;394:155-161.

2. Guerrero-Beltrán CE, Calderón-Oliver M, Pedraza-Chaverri J, Chirino YI. Protective effect of sulforaphane against oxidative stress: recent advances. Exp Toxicol Pathol. 2012;64:503-508.

3. Li N, Nel AE. Role of the Nrf2-mediated signaling pathway as a negative regulator of inflammation: implications for the impact of particulate pollutants on asthma. Antioxid Redox Signal. 2006;8:88-98.

4. Zhang X, Xiao Z, Yao J, Zhao G, Fa X, Niu J. Participation of protein kinase $\mathrm{C}$ in the activation of $\mathrm{Nrf} 2$ signaling by ischemic preconditioning in the isolated rabbit heart. Mol Cell Biochem. 2013;372:169-179.

5. Alfieri A, Srivastava S, Siow RC, et al. Sulforaphane preconditioning of the Nrf2/HO-1 defense pathway protects the cerebral vasculature against blood-brain barrier disruption and neurological deficits in stroke. Free Radic Biol Med. 2013;65:1012-1022.

6. Zhao X, Sun G, Zhang J, et al. Transcription factor nrf2 protects the brain from damage produced by intracerebral hemorrhage. Stroke. 2007;38: 3280-3286.

7. Deinsberger W, Lang C, Hornig C, Boeker DK. Stereotactic aspiration and fibrinolysis of spontaneous supratentorial intracerebral hematomas versus conservative treatment: a matched-pair study. Zentralbl Neurochir. 2003;64:145-150.

8. Garcia JH, Wagner S, Liu KF, Hu XJ. Neurological deficit and extent of neuronal necrosis attributable to middle cerebral artery occlusion in rats. Statistical validation. Stroke. 1995;26:627-634; discussion 635.

9. Steel R, Cowan J, Payerne E, O'Connell MA, Searcey M. Antiinflammatory Effect of a Cell-Penetrating Peptide Targeting the Nrf2/ Keap1 Interaction. ACS Med Chem Lett. 2012;3:407-410.

10. Graber DJ, Hickey WF, Stommel EW, Harris BT. Anti-inflammatory efficacy of dexamethasone and Nrf2 activators in the CNS using brain slices as a model of acute injury. J Neuroimmune Pharmacol. 2012;7: 266-278.

11. Lee IS, Lim J, Gal J, et al. Anti-inflammatory activity of xanthohumol involves heme oxygenase-1 induction via NRF2-ARE signaling in microglial BV2 cells. Neurochem Int. 2011;58:153-160.

12. Huang XS, Chen HP, Yu HH, Yan YF, Liao ZP, Huang QR. Nrf2dependent upregulation of antioxidative enzymes: a novel pathway for hypoxic preconditioning-mediated delayed cardioprotection. Mol Cell Biochem. 2014;385:33-41.

13. Khodagholi F, Tusi SK. Stabilization of Nrf2 by tBHQ prevents LPSinduced apoptosis in differentiated PC12 cells. Mol Cell Biochem. 2011;354:97-112. 
14. Shah ZA, Li RC, Ahmad AS, et al. The flavanol (-)-epicatechin prevents stroke damage through the $\mathrm{Nrf2/HO1}$ pathway. J Cereb Blood Flow Metab. 2010;30:1951-1961.

15. Zhang Z, Cui W, Li G, et al. Baicalein protects against 6-OHDAinduced neurotoxicity through activation of Keap1/Nrf2/Ho-1 and involving PKCA and PI3K/AKT signaling pathways. J Agric Food Chem. 2012;60:8171-8182.

16. Chen L, Wang L, Zhang X, et al. The protection by octreotide against experimental ischemic stroke: up-regulated transcription factor Nrf2, HO-1 and down-regulated NF- $\kappa \mathrm{B}$ expression. Brain Res. 2012;1475: $80-87$.

17. Sahin K, Tuzcu M, Orhan C, et al. The effects of chromium picolinate and chromium histidinate administration on NF- $\mathrm{\kappa B}$ and $\mathrm{Nrf} 2 / \mathrm{HO}-1$ pathway in the brain of diabetic rats. Biol Trace Elem Res. 2012;150: 291-296.
18. Buhrmann C, Mobasheri A, Busch F, et al. Curcumin modulates nuclear factor kappaB (NF-kappaB)-mediated inflammation in human tenocytes in vitro: role of the phosphatidylinositol 3-kinase/Akt pathway. $J$ Biol Chem. 2011;286:28556-28566.

19. Prabhavathy D, Prabhakar BN, Karunagaran D. HPV16 E2-mediated potentiation of NF- $\kappa \mathrm{B}$ activation induced by TNF- $\alpha$ involves parallel activation of STAT3 with a reduction in E2-induced apoptosis. Mol Cell Biochem. 2014;394:77-90. 


\section{Supplementary material}

A
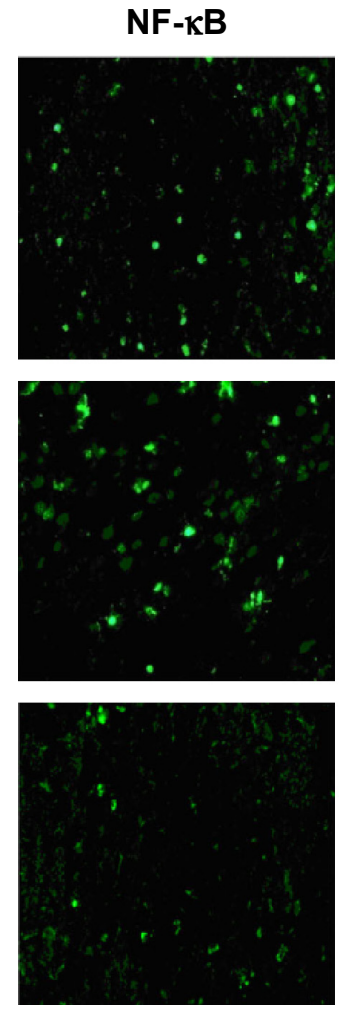

B
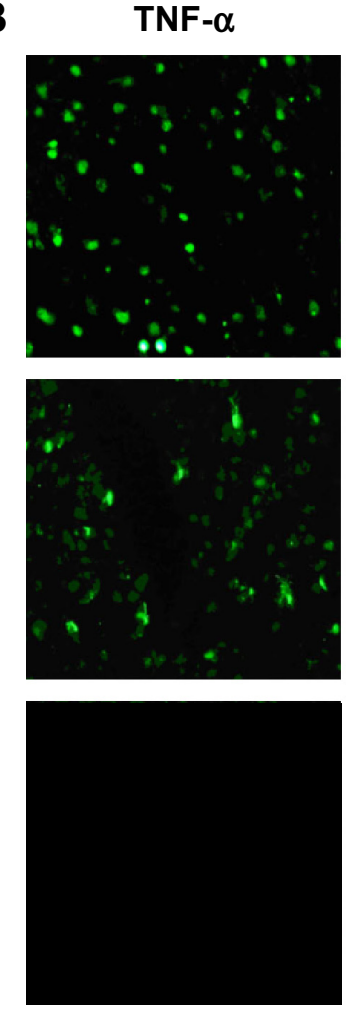

Nrf2
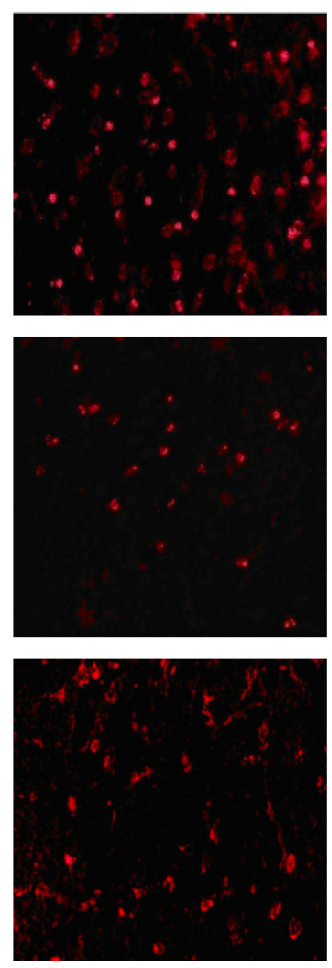

Nrf2
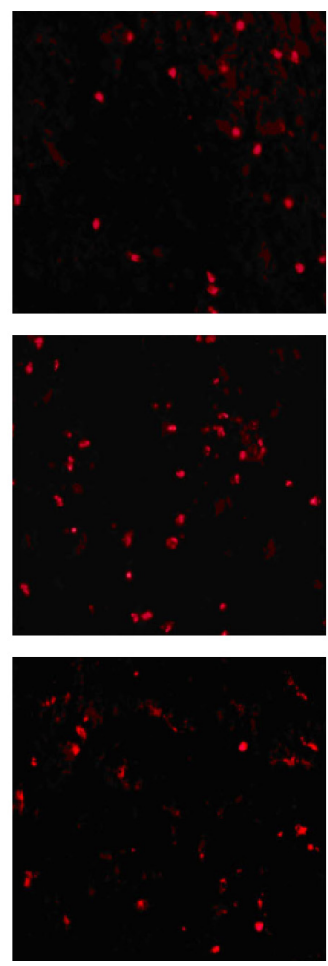

DAPI
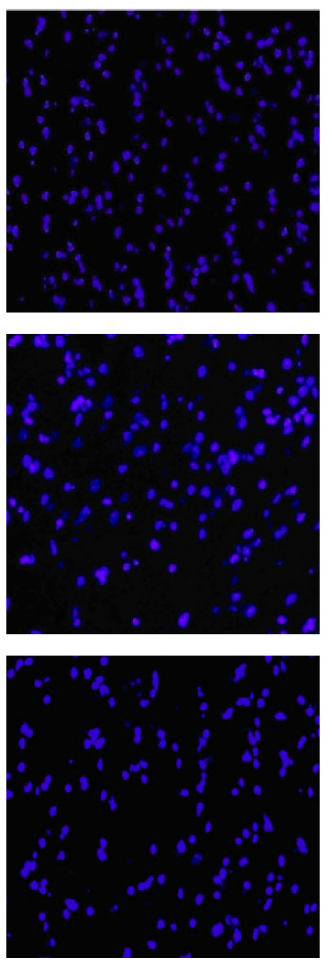

DAPI
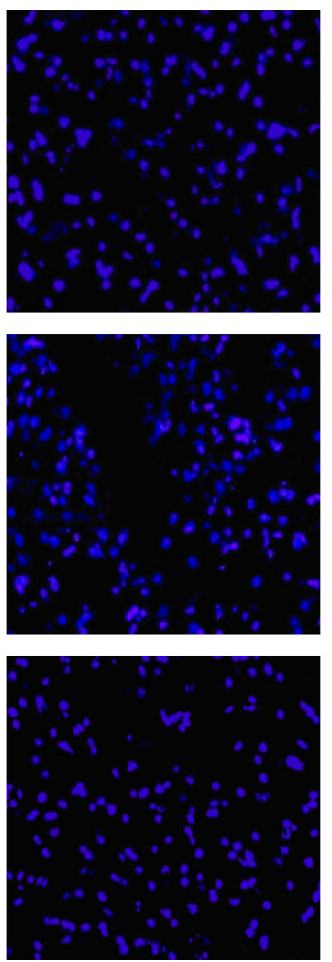

Merge

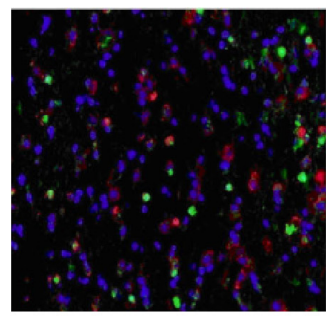

$\mathrm{ICH}$

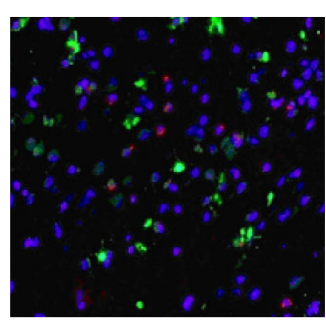

RA

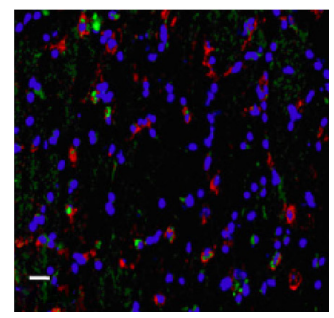

SFN

$\mathrm{ICH}$

RA

SFN

Figure SI Cells stained positive for both Nrf2 and NF- $\kappa B$ (or TNF- $\alpha$ ) protein.

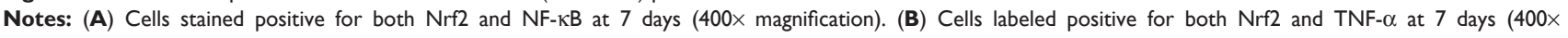
magnification).

Abbreviations: DAPI, 4',6-diamidino-2-phenylindole; ICH, intracerebral hemorrhage; RA, retinoic acid; SFN, sulforaphane; NF- $\kappa B$, nuclear factor- $\kappa B$; TNF- $\alpha$, tumor necrosis factor- $\alpha$; Nrf2, nuclear factor erythroid 2-related factor 2 . 


\section{Publish your work in this journal}

Drug Design, Development and Therapy is an international, peerreviewed open-access journal that spans the spectrum of drug design and development through to clinical applications. Clinical outcomes, patient safety, and programs for the development and effective, safe, and sustained use of medicines are a feature of the journal, which

has also been accepted for indexing on PubMed Central. The manuscript management system is completely online and includes a very quick and fair peer-review system, which is all easy to use. Visit http://www.dovepress.com/testimonials.php to read real quotes from published authors.

Submit your manuscript here: http://www.dovepress.com/drug-design-development-and-therapy-journal 